\title{
On $p$-almost direct products and residual properties of pure braid groups of nonorientable surfaces
}

\author{
PAOLO BELLINGERI \\ SYLVAIN GERVAIS
}

\begin{abstract}
We prove that the $n^{\text {th }}$ pure braid group of a nonorientable surface (closed or with boundary, but different from $\mathbb{R P}^{2}$ ) is residually 2-finite. Consequently, this group is residually nilpotent. The key ingredient in the closed case is the notion of $p$-almost direct product, which is a generalization of the notion of almost direct product. We also prove some results on lower central series and augmentation ideals of $p$-almost direct products.
\end{abstract}

20F14, 20F36, 57M05; 20D15

\section{Introduction}

Let $M$ be a compact, connected surface (orientable or not, possibly with boundary) and $F_{n}(M)=\left\{\left(x_{1}, \ldots, x_{n}\right) \in M^{n} \mid x_{i} \neq x_{j}\right.$ for $\left.i \neq j\right\}$ its $n^{\text {th }}$ configuration space. The fundamental group $\pi_{1}\left(F_{n}(M)\right)$ is called the $n^{\text {th }}$ pure braid group of $M$ and shall be denoted by $P_{n}(M)$.

The mapping class group $\Gamma(M)$ of $M$ is the group of isotopy classes of homeomorphisms $h: M \rightarrow M$ which act as the identity on the boundary. Let $\mathcal{X}_{n}=\left\{z_{1}, \ldots, z_{n}\right\}$ be a set of $n$ distinguished points in the interior of $M$; the pure mapping class group $\mathrm{P} \Gamma\left(M, \mathcal{X}_{n}\right)$ relative to $\mathcal{X}_{n}$ is the group of isotopy classes of homeomorphisms $h: M \rightarrow M$ satisfying $h\left(z_{i}\right)=z_{i}$ for all $i$ : since this group does not depend on the choice of the set $\mathcal{X}_{n}$ but only on its cardinality we can write $\mathrm{P}_{n} \Gamma(M)$ instead of $\mathrm{P} \Gamma\left(M, \mathcal{X}_{n}\right)$. Forgetting the marked points, we get a morphism $\mathrm{P}_{n} \Gamma(M) \rightarrow \Gamma(M)$ whose kernel is known to be isomorphic to $P_{n}(M)$ when $M$ is not a sphere, a torus, a projective plane or a Klein bottle (see Scott [28] and Guaschi and Juan-Pineda [20]).

Now, recall that if $\mathcal{P}$ is a group-theoretic property, then a group $G$ is said to be residually $\mathcal{P}$ if, for all $g \in G, g \neq 1$, there exists a group homomorphism $\varphi: G \rightarrow H$ such that $H$ satisfies $\mathcal{P}$ and $\varphi(g) \neq 1$. We are interested in the following properties: to be nilpotent, to be free and to be a finite $p$-group for a prime number $p$ (mostly $p=2$ ). Recall that, if for subgroups $H$ and $K$ of $G,[H, K]$ is the subgroup generated 
by $\{[h, k] \mid(h, k) \in H \times K\}$ where $[h, k]=h^{-1} k^{-1} h k$, the lower central series of $G$, $\left(\Gamma_{k} G\right)_{k \geq 1}$, is defined inductively by $\Gamma_{1} G=G$ and $\Gamma_{k+1} G=\left[G, \Gamma_{k} G\right]$. It is well known that $G$ is residually nilpotent if and only if $\bigcap_{k=1}^{\infty} \Gamma_{k} G=\{1\}$. From the lower central series of $G$ one can define another filtration $D_{1}(G) \supseteq D_{2}(G) \supseteq \cdots$ setting $D_{1}(G)=G$, and for $i \geq 2$ defining

$$
D_{i}(G)=\left\{x \in G \mid \exists n \in \mathbb{N}^{*} \text { with } x^{n} \in \Gamma_{i}(G)\right\} .
$$

After Garoufalidis and Levine [13], this filtration is called the rational lower central series of $G$, and a group $G$ is residually torsion-free nilpotent if and only if $\bigcap_{i=1}^{\infty} D_{i}(G)=\{1\}$.

When $M$ is an orientable surface of positive genus (possibly with boundary) or a disc with holes, it is proved in Bellingeri, Gervais and Guaschi [6] and Bardakov and Bellingeri [1] that $P_{n}(M)$ is residually torsion-free nilpotent for all $n \geq 1$. The fact that a group is residually torsion-free nilpotent has several important consequences, notably that the group is bi-orderable (see Botto Mura and Rhemtulla [8]) and residually $p$-finite (see Gruenberg [19]). The goal of this article is to study the nonorientable case and, more precisely, to prove the following:

Theorem 1.1 The $n^{\text {th }}$ pure braid group of a nonorientable surface different from $\mathbb{R P}^{2}$ is residually $2-$ finite.

In the case of $P_{n}\left(\mathbb{R P}^{2}\right)$ we give some partial results at the end of Section 4 . Since a finite 2 -group is nilpotent, a residually 2 -finite group is residually nilpotent. Thus, we have:

Corollary 1.2 The $n^{\text {th }}$ pure braid group of a nonorientable surface different from $\mathbb{R P}^{2}$ is residually nilpotent.

In González-Meneses [17] it was shown that the $n^{\text {th }}$ pure braid group of a nonorientable surface is not bi-orderable and therefore it is not residually torsion-free nilpotent. Our technique doesn't extend to $p \neq 2$; therefore the question if pure braid groups of nonorientable surfaces are residually $p$ for some $p \neq 2$ is still open (recall that there are groups residually $p$ for infinitely many primes $p$ which are not residually torsion-free nilpotent; see Hartley [21]).

One can prove that finite-type invariants separate classical braids using the fact that the pure braid group $P_{n}$ is residually nilpotent without torsion (see Papadima [25]). Moreover, using the residual properties discussed above it is possible to construct algebraically a universal finite-type invariant over $\mathbb{Z}$ for the classical braid group $B_{n}$ (see [25]). Similar constructions were afterwards proposed for braids on orientable 
surfaces (see Bellingeri and Funar [5] and González-Meneses and Paris [18]): in a further paper we will explore the relevance of Theorem 1.1 in the realm of finite-type invariants over $\mathbb{Z} / 2 \mathbb{Z}$ for braids on nonorientable surfaces.

From now on, $M=N_{g, b}$ is a nonorientable surface of genus $g$ with $b$ boundary components, simply denoted by $N_{g}$ when $b=0$. We will see $N_{g}$ as a sphere $S^{2}$ with $g$ open discs removed and $g$ Möbius strips glued on each circle (see Figure 4, where each crossed disc represents a Möbius strip). The surface $N_{g, b}$ is obtained from $N_{g}$ by removing $b$ open discs. The mapping class groups $\Gamma\left(N_{g, b}\right)$ and pure mapping class group $P_{n} \Gamma\left(N_{g, b}\right)$ will be denoted by $\Gamma_{g, b}$ and $\Gamma_{g, b}^{n}$, respectively.

The paper is organized as follows. In Section 2, we prove Theorem 1.1 for surfaces with boundary: following what the authors did in the orientable case (see [6]), we embed $P_{n}\left(N_{g, b}\right)$ in a Torelli group. The difference here is that we must consider mod 2 Torelli groups. In Section 3 we introduce the notion of $p$-almost direct product, which generalizes the notion of almost direct product (see Definition 3.1) and we prove some results on lower central series and augmentation ideals of $p$-almost direct products (Theorems 3.3 and 3.6) that can be compared with similar results on almost direct products (Theorem 3.1 in Falk and Randell [12] and Theorem 3.1 in Papadima [25]).

In Section 4, the existence of a split exact sequence

$$
1 \longrightarrow P_{n-1}\left(N_{g, 1}\right) \longrightarrow P_{n}\left(N_{g}\right) \longrightarrow \pi_{1}\left(N_{g}\right) \longrightarrow 1
$$

and results from Sections 2 and 3 are used to prove Theorem 1.1 in the closed case (Theorem 4.5). The method is similar to the one developed for orientable surfaces in [1]: the difference will be that the semi-direct product $P_{n-1}\left(N_{g, 1}\right) \rtimes \pi_{1}\left(N_{g}\right)$ is a 2-almost-direct product (and not an almost-direct product as in the case of closed oriented surfaces).

Acknowledgments The research of the first author was partially supported by French grant ANR-11-JS01-002-01. The authors are grateful to Carolina de Miranda e Pereiro and John Guaschi for useful discussions and comments and to the anonymous referee for helpful remarks, in particular on a previous version of Proposition 4.3.

\section{The case of non-empty boundary}

In this section, $N=N_{g, b}$ is a nonorientable surface of genus $g \geq 1$ with boundary (ie $b \geq 1)$. In this case, one has $P_{n}(N)=\operatorname{Ker}\left(\Gamma_{g, b}^{n} \rightarrow \Gamma_{g, b}\right)$ for all $n \geq 1$. 


\subsection{Notation}

We will follow notation from [27]. A simple closed curve in $N$ is an embedding $\alpha: S^{1} \rightarrow N \backslash \partial N$; with a usual abuse of notation, we will call the image of a simple closed curve a simple closed curve also. Such a curve is said to be two-sided or one-sided if it admits a regular neighborhood homeomorphic to an annulus or a Möbius strip, respectively. We shall consider the following elements in $\Gamma_{g, b}$ :

- If $\alpha$ is a two-sided simple closed curve in $N$ with a given orientation, $\tau_{\alpha}$ is a Dehn twist along $\alpha$.

- Let $\mu$ and $\alpha$ be two simple closed curves such that $\mu$ is one-sided, $\alpha$ is oriented and two-sided, and such that $|\alpha \cap \mu|=1$. A regular neighborhood $K$ of $\alpha \cup \mu$ is diffeomorphic to a Klein bottle with one hole, and a regular neighborhood $M$ of $\mu$ is diffeomorphic to a Möbius strip. Pushing $M$ once along $\alpha$, we get a diffeomorphism of $K$ fixing the boundary (see Figure 1): it can be extended to $N$ by the identity. Such a diffeomorphism is called a crosscap slide, and denoted by $Y_{\mu, \alpha}$.

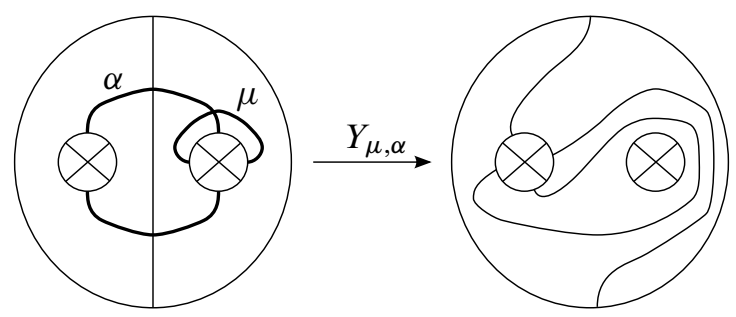

Figure 1: Crosscap slide.

\subsection{Blowup homomorphism}

Here we recall the construction of the blowup homomorphism $\eta_{g, b}^{n}: \Gamma_{g, b}^{n} \rightarrow \Gamma_{g+n, b}$ given in [30; 31] and [27].

Let $U=\{z \in \mathbb{C}|| z \mid \leq 1\}$ and, for $i=1, \ldots, n$, fix an embedding $e_{i}: U \rightarrow N$ such that $e_{i}(0)=z_{i}, e_{i}(U) \cap e_{j}(U)=\varnothing$ if $i \neq j$ and $e_{i}(U) \cap \partial N=\varnothing$ for all $i$. If we remove the interior of each $e_{i}(U)$ (thus getting the surface $N_{g, b+n}$ ) and identify, for each $z \in \partial U, e_{i}(z)$ with $e_{i}(-z)$, we get a nonorientable surface of genus $g+n$ with $b$ boundary components, that is to say a surface homeomorphic to $N_{g+n, b}$. Let us denote by $\gamma_{i}=e_{i}\left(S^{1}\right)$ the boundary of $e_{i}(U)$, and by $\mu_{i}$ its image in $N_{g+n, b}$; it is a one-sided simple closed curve.

Now, let $h$ be an element of $\Gamma_{g, b}^{n}$. It can be represented by a homeomorphism $N_{g, b} \rightarrow N_{g, b}$, still denoted $h$, such that: 
(1) $h\left(e_{i}(z)\right)=e_{i}(z)$ if $h$ preserves local orientation at $z_{i}$.

(2) $h\left(e_{i}(z)\right)=e_{i}(\bar{z})$ if $h$ reverses local orientation at $z_{i}$.

Such a homeomorphism $h$ commutes with the identification leading to $N_{g+n, b}$ and thus induces an element $\eta(h) \in \Gamma_{g+n, b}$. It is proved in [31] that the map

$$
\eta_{g, b}^{n}=\eta: \Gamma_{g, b}^{n} \rightarrow \Gamma_{g+n, b}, \quad h \longmapsto \eta(h)
$$

is well defined for $n=1$, but the proof also works for $n>1$. This homomorphism is called the blowup homomorphism.

Proposition 2.1 The blowup homomorphism $\eta_{g, b}^{n}: \Gamma_{g, b}^{n} \rightarrow \Gamma_{g+n, b}$ is injective if $(g+n, b) \neq(2,0)$.

Remark 2.2 This result is proved in [30] for $(g, b)=(0,1)$, but the proof can be adapted in our case as follows.

Proof Suppose that $h: N_{g, b} \rightarrow N_{g, b}$ is a homeomorphism satisfying $h\left(z_{i}\right)=z_{i}$ for all $i$ and that $\eta(h): N_{g+n, b} \rightarrow N_{g+n, b}$ is isotopic to the identity. Then $h$ is isotopic to a map equal to the identity on $e_{i}(U)$ for all $i$. If not, $h$ reverses local orientation at $z_{i}$ and $h\left(\gamma_{i}\right)$ is isotopic to $\gamma_{i}^{-1}$. Then $\eta(h)\left(\gamma_{i}\right)$ is isotopic to $\mu_{i}$ and $\mu_{i}^{-1}$ and we get $2\left[\mu_{i}\right]=0$ in $H_{1}\left(N_{g+n, b} ; \mathbb{Z}\right)$, which is a contradiction. Consequently, $h$ lies in the kernel of the natural map $\Gamma_{g, b+n} \rightarrow \Gamma_{g+n, b}$ induced by gluing a Möbius strip onto $n$ boundary components. However, this kernel is generated by the Dehn twists along the curves $\gamma_{i}$ (see [29, Theorem $\left.3.6^{1}\right]$ ). Now, any $\gamma_{i}$ bounds a disc with one marked point in $N_{g, b}$ : the corresponding Dehn twist is trivial in $\Gamma_{g, b}$ and therefore $h$ is isotopic to the identity.

\subsection{Embedding $P_{n}\left(N_{g, b}\right)$ in $\Gamma_{g+n+2(b-1), 1}$}

Since $b \geq 1$, we'll view $N_{g, b}$ as a disc $D^{2}$ with $g+b-1$ open discs removed and $g$ Möbius strips glued on $g$ boundary components so obtained (see Figure 2).

Proposition 2.3 For $g \geq 1, b \geq 1$ and $n \geq 1, P_{n}\left(N_{g, b}\right)$ has the following complete set of generators (depicted in Figures 2 and 4):

$$
\left(B_{i, j}\right)_{1 \leq i<j \leq n}, \quad\left(\rho_{k, l}\right)_{\substack{1 \leq k \leq n \\ 1 \leq l \leq g}} \quad \text { and } \quad\left(x_{u, t}\right)_{\substack{1 \leq u \leq n \\ 1 \leq t \leq b-1}} .
$$

${ }^{1}$ This result is wrong when $(g+n, b)=(2,0)$. 


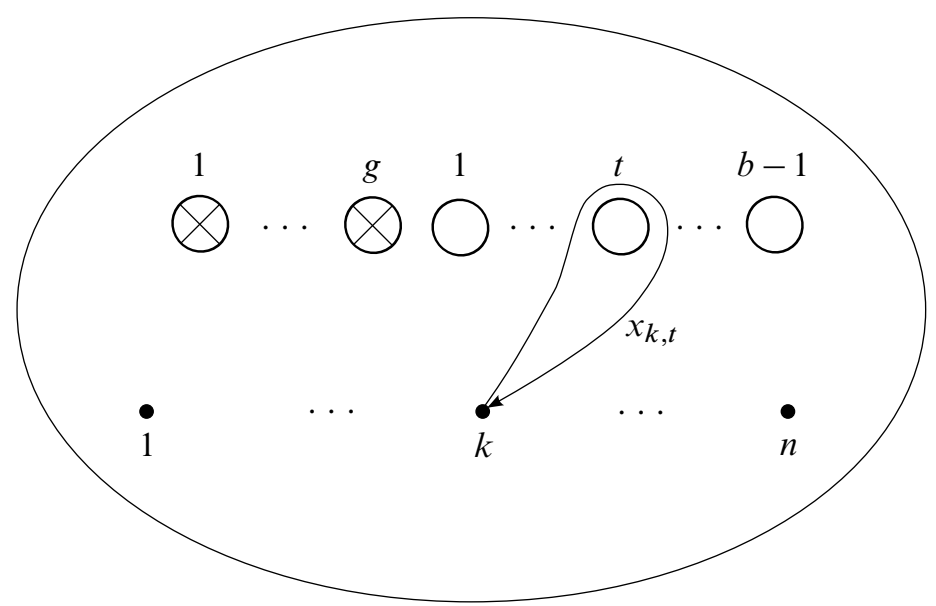

Figure 2: Generators $x_{k, t}$ for $P_{n}\left(N_{g, b}\right), b \geq 1$. See Figure 4 for a picture of generators $B_{i, j}$ and $\rho_{k, l}$.

Proof The proof works by induction and generalizes those of [16] (closed nonorientable case) and [4] (orientable case, possibly with boundary components). It uses the following short exact sequence obtained by forgetting the last strand (see [11]):

$$
1 \longrightarrow \pi_{1}\left(N_{g, b} \backslash\left\{z_{1}, \ldots, z_{n}\right\}, z_{n+1}\right) \stackrel{\alpha}{\longrightarrow} P_{n+1}\left(N_{g, b}\right) \stackrel{\beta}{\longrightarrow} P_{n}\left(N_{g, b}\right) \longrightarrow 1 .
$$

The set of generators is complete for $n=1: P_{1}\left(N_{g, b}\right)=\pi_{1}\left(N_{g, b}\right)$ is free on the $\rho_{1, l}$ and $x_{1, t}$ for $1 \leq l \leq g$ and $1 \leq t \leq b-1$. Suppose inductively that $P_{n}\left(N_{g, b}\right)$ has the given complete set of generators. Then observe that

$$
\left\{B_{i, n+1} \mid 1 \leq i \leq n\right\} \cup\left\{\rho_{n+1, l} \mid 1 \leq l \leq g\right\} \cup\left\{x_{n+1, t} \mid 1 \leq t \leq b-1\right\}
$$

is a free generators set of $\operatorname{Im}(\alpha)$ and

$$
\left(B_{i, j}\right)_{1 \leq i<j \leq n}, \quad\left(\rho_{k, l}\right)_{\substack{1 \leq k \leq n \\ 1 \leq l \leq g}} \quad \text { and } \quad\left(x_{u, t}\right)_{\substack{1 \leq u \leq n \\ 1 \leq t \leq b-1}}
$$

are coset representatives for the considered generators of $P_{n}\left(N_{g, b}\right)$; this is a complete set of generators for $P_{n+1}\left(N_{g, b}\right)$; see for instance [22, Theorem 1, Chapter 13]. Let us also remark that the above exact sequence could be used, as in [4] and [16], to find a complete set of relations for the group $P_{n}\left(N_{g, b}\right)$.

Gluing a one-holed torus onto $b-1$ boundary components of $N_{g, b}$ (recall that $b \geq 1$ in this second section), we get $N_{g, b}$ as a subsurface of $N_{g+2(b-1), 1}$. This inclusion induces a homomorphism $\chi_{g, b}: \Gamma_{g, b} \rightarrow \Gamma_{g+2(b-1), 1}$ which is injective (see [29]). 
Thus, the composed map

$$
\lambda_{g, b}^{n}=\chi_{g+n, b} \circ \eta_{g, b}^{n}: \Gamma_{g, b}^{n} \rightarrow \Gamma_{g+n+2(b-1), 1}
$$

is also injective.

Recall that the mod $p$ Torelli group $I_{p}\left(N_{g, 1}\right)$ is the subgroup of $\Gamma_{g, 1}$ defined as the kernel of the action of $\Gamma_{g, 1}$ on $H_{1}\left(N_{g, 1} ; \mathbb{Z} / p \mathbb{Z}\right)$. In the following we will consider in particular the case of the mod 2 Torelli group $I_{2}\left(N_{g, 1}\right)$.

Proposition 2.4 If $b \geq 1, \lambda_{g, b}^{n}\left(P_{n}\left(N_{g, b}\right)\right)$ is a subgroup of the mod 2 Torelli subgroup $I_{2}\left(N_{g+n+2(b-1), 1}\right)$.

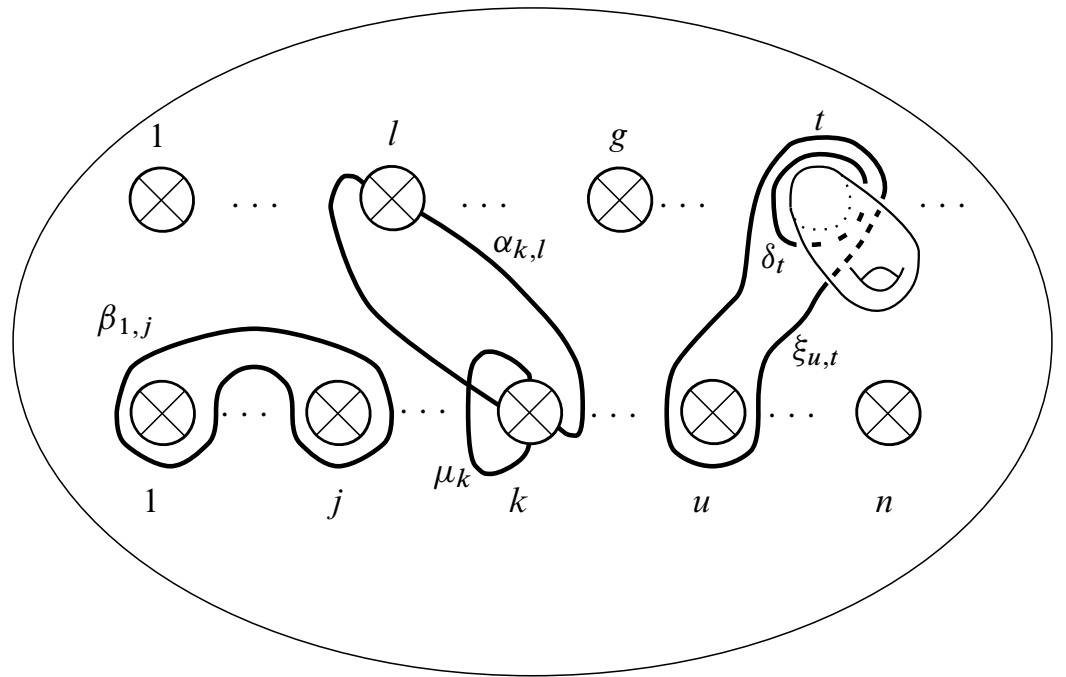

Figure 3: Image of the generators of $P_{n}\left(N_{g, b}\right)$ in $\Gamma_{g+n+2(b-1), 1}$.

Proof The image of the generators (see Figures 2, 4 and Proposition 2.3)

$$
\left(B_{i, j}\right)_{1 \leq i<j \leq n}, \quad\left(\rho_{k, l}\right)_{\substack{1 \leq k \leq n \\ 1 \leq l \leq g}} \quad \text { and } \quad\left(x_{u, t}\right)_{\substack{1 \leq u \leq n \\ 1 \leq t \leq b-1}}
$$

of $P_{n}\left(N_{g, b}\right)$ under $\lambda_{g, b}^{n}$ are, respectively (see Figure 3):

- Dehn twists along curves $\beta_{i, j}$ which bound a subsurface homeomorphic to $N_{2,1}$.

- Crosscap slides $Y_{\mu_{k}, \alpha_{k, l}}$.

- The product $\tau_{\xi_{u, t}} \tau_{\delta_{t}}^{-1}$ of Dehn twists along the bounding curves $\xi_{u, t}$ and $\delta_{t}$.

According to [31], all of these elements are in $I_{2}\left(N_{g+n+2(b-1), 1}\right)$. 
Remark 2.5 The embedding from Proposition 2.4 is invalid for $I_{p}\left(N_{g+n+2(b-1), 1}\right)$ when $p \neq 2$ : for example, the crosscap slide $Y_{\mu_{k}, \alpha_{k, l}}$ is not in the $\bmod p$ Torelli subgroup since it sends $\mu_{k}$ to $\mu_{k}^{-1}$.

\subsection{Conclusion of the proof}

We shall use the following result, which is a straightforward consequence of a similar result for mod $p$ Torelli groups of orientable surfaces due to L Paris [26]:

Theorem 2.6 Let $g \geq 1$. The $\bmod p$ Torelli group $I_{p}\left(N_{g, 1}\right)$ is residually $p$-finite.

Proof We use the Dehn-Nielsen-Baer theorem (see for instance [32, Theorem 5.15.3]), which states that $\Gamma_{g, 1}$ embeds in $\operatorname{Aut}\left(\pi_{1}\left(N_{g, 1}\right)\right)$. Since $\pi_{1}\left(N_{g, 1}\right)$ is free we can apply [26, Theorem 1.4] which claims that, if $G$ is a free group, its $\bmod p$ Torelli group (ie the kernel of the canonical map from $\operatorname{Aut}(G)$ to $\operatorname{GL}\left(H_{1}\left(G, \mathbb{F}_{p}\right)\right)$ is residually $p$ finite. Therefore $I_{p}\left(N_{g, 1}\right)$ is residually $p$-finite.

Theorem 2.7 Let $g \geq 1, b>0, n \geq 1$. Then $P_{n}\left(N_{g, b}\right)$ is residually 2-finite.

Proof The group $P_{n}\left(N_{g, b}\right)$ is a subgroup of $I_{2}\left(N_{g+n+2(b-1), 1}\right)$ by Proposition 2.4 and by injectivity of the map $\lambda_{g, b}^{n}$. Then by Theorem 2.6 it follows that $P_{n}\left(N_{g, b}\right)$ is residually 2 -finite.

\section{3 p-almost direct products}

\subsection{On residually $p$-finite groups}

Let $p$ be a prime number and $G$ a group. If $H$ is a subgroup of $G$, we denote by $H^{p}$ the subgroup generated by $\left\{h^{p} \mid h \in H\right\}$. Following [26], we define the lower $\mathbb{F}_{p}$-linear central filtration $\left(\gamma_{n}^{p} G\right)_{n \in \mathbb{N}^{*}}$ of $G$ as follows: $\gamma_{1}^{p} G=G$ and, for $n \geq 1, \gamma_{n+1}^{p} G$ is the subgroup of $G$ generated by $\left[G, \gamma_{n}^{p} G\right] \cup\left(\gamma_{n}^{p} G\right)^{p}$. Note that the subgroups $\gamma_{n}^{p} G$ are characteristic in $G$ and that the quotient group $G / \gamma_{2}^{p} G$ is nothing but the first homology group $H_{1}\left(G ; \mathbb{F}_{p}\right)$. The following are proved in [26]:

- $\left[\gamma_{m}^{p} G, \gamma_{n}^{p} G\right] \subset \gamma_{m+n}^{p} G$ for $m, n \geq 1$.

- A finitely generated group $G$ is a finite $p$-group if and only if there exists some $N \geq 1$ such that $\gamma_{N}^{p} G=\{1\}$.

- A finitely generated group $G$ is residually $p$-finite if and only if $\bigcap_{n=1}^{\infty} \gamma_{n}^{p} G=\{1\}$. Clearly, if $f: G \rightarrow G^{\prime}$ is a group homomorphism, then $f\left(\gamma_{n}^{p} G\right) \subset \gamma_{n}^{p} G^{\prime}$ for all $n \geq 1$. 
Definition 3.1 Let

$$
1 \longrightarrow A \hookrightarrow B \underset{\sigma \ldots \ldots}{\longrightarrow} C \longrightarrow 1
$$

be a split exact sequence.

- If the action of $C$ induced on $H_{1}(A ; \mathbb{Z})$ is trivial (ie the action is trivial on $\left.A^{\mathrm{Ab}}=A /[A, A]\right)$, we say that $\mathrm{B}$ is an almost direct product of $A$ and $C$.

- If the action of $C$ induced on $H_{1}\left(A ; \mathbb{F}_{p}\right)$ is trivial (ie the action is trivial on $A / \gamma_{2}^{p} A$ ), we say that $B$ is a $p$-almost direct product of $A$ and $C$.

Let us remark that, as in the case of almost direct products [7, Proposition 6.3], the property of being a $p$-almost direct product does not depend on the choice of section.

Proposition 3.2 Let $1 \longrightarrow A \hookrightarrow B \stackrel{\lambda}{\longrightarrow} C \longrightarrow 1$ be a split exact sequence of groups. Let $\sigma, \sigma^{\prime}$ be sections for $\lambda$, and suppose that the induced action of $C$ on $A$ via $\sigma$ on $H_{1}\left(A ; \mathbb{F}_{p}\right)$ is trivial. Then the same is true for the section $\sigma^{\prime}$.

Proof Let $a \in A$ and $c \in C$. By hypothesis, $\sigma(c) a(\sigma(c))^{-1} \equiv a \bmod \gamma_{2}^{p} A$. Since $\sigma^{\prime}$ is also a section for $\lambda$, we have $\lambda \circ \sigma^{\prime}(c)=\lambda \circ \sigma(c)$, and so $\sigma^{\prime}(c)(\sigma(c))^{-1} \in \operatorname{Ker}(\lambda)$. Thus there exists $a^{\prime} \in A$ such that $\sigma^{\prime}(c)=a^{\prime} \sigma(c)$, and hence

$$
\sigma^{\prime}(c) a\left(\sigma^{\prime}(c)\right)^{-1} \equiv a^{\prime} \sigma(c) a(\sigma(c))^{-1} a^{\prime-1} \equiv a^{\prime} a a^{\prime-1} \equiv a \bmod \gamma_{2}^{p} A .
$$

Thus the induced action of $C$ on $H_{1}\left(A ; \mathbb{F}_{p}\right)$ via $\sigma^{\prime}$ is also trivial.

The first goal of this section is to prove the following theorem (see [12, Theorem 3.1] for an analogous result for almost direct products).

Theorem 3.3 Let

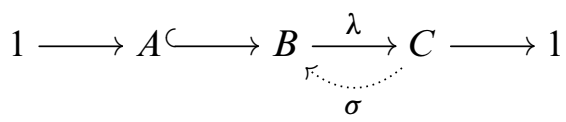

be a split exact sequence where $B$ is a $p$-almost direct product of $A$ and $C$. Then, for all $n \geq 1$, one has a split exact sequence

$$
1 \longrightarrow \gamma_{n}^{p} A \longleftrightarrow \gamma_{n}^{p} B \underset{\sigma_{n}}{\stackrel{\lambda_{n}}{\longrightarrow}} \gamma_{n}^{p} C \longrightarrow 1,
$$

where $\lambda_{n}$ and $\sigma_{n}$ are restrictions of $\lambda$ and $\sigma$. 
We shall need the following preliminary result.

Lemma 3.4 Under the hypotheses of Theorem 3.3, one has

$$
\left[\gamma_{m}^{p} C^{\prime}, \gamma_{n}^{p} A\right] \subset \gamma_{m+n}^{p} A \text { for all } m, n \geq 1,
$$

where $C^{\prime}$ denotes $\sigma(C)$.

Proof First, we prove by induction on $n$ that $\left[C^{\prime}, \gamma_{n}^{p} A\right] \subset \gamma_{n+1}^{p} A$ for all $n \geq 1$. The case $n=1$ corresponds to the hypotheses: the action of $C$ on $H_{1}\left(A ; \mathbb{F}_{p}\right)=A / \gamma_{2}^{p} A$ is trivial if and only if $\left[C^{\prime}, A\right] \subset \gamma_{2}^{p} A$. Thus, suppose that $\left[C^{\prime}, \gamma_{n}^{p} A\right] \subset \gamma_{n+1}^{p} A$ for some $n \geq 1$ and let us prove that $\left[C^{\prime}, \gamma_{n+1}^{p} A\right] \subset \gamma_{n+2}^{p} A$. In view of the definition of $\gamma_{n+1}^{p} A$, we have to prove that

$$
\left[C^{\prime},\left[A, \gamma_{n}^{p} A\right]\right] \subset \gamma_{n+2}^{p} A \text { and }\left[C^{\prime},\left(\gamma_{n}^{p} A\right)^{p}\right] \subset \gamma_{n+2}^{p} A
$$

For the first case, we use a classical result (see [24, Theorem 5.2]) which says

$$
\left[C^{\prime},\left[A, \gamma_{n}^{p} A\right]\right]=\left[\gamma_{n}^{p} A,\left[C^{\prime}, A\right]\right]\left[A,\left[\gamma_{n}^{p} A, C^{\prime}\right]\right] .
$$

We have just seen that $\left[C^{\prime}, A\right] \subset \gamma_{2}^{p} A$, thus

$$
\left[\gamma_{n}^{p} A,\left[C^{\prime}, A\right]\right] \subset\left[\gamma_{n}^{p} A, \gamma_{2}^{p} A\right] \subset \gamma_{n+2}^{p} A .
$$

Then, the induction hypotheses says that $\left[\gamma_{n}^{p} A, C^{\prime}\right] \subset \gamma_{n+1}^{p} A$, thus

$$
\left[A,\left[\gamma_{n}^{p} A, C^{\prime}\right]\right] \subset\left[A, \gamma_{n+1}^{p} A\right] \subset \gamma_{n+2}^{p} A .
$$

The second case works as follows: for $c \in C^{\prime}$ and $x \in \gamma_{n}^{p} A$, one has, using the fact that $[u, v w]=[u, w][u, v][[u, v], w]($ see $[24])$,

$$
\begin{aligned}
{\left[c, x^{p}\right] } & =[c, x]\left[c, x^{p-1}\right]\left[\left[c, x^{p-1}\right], x\right] \\
& =\cdots=[c, x]^{p}[[c, x], x]\left[\left[c, x^{2}\right], x\right] \cdots\left[\left[c, x^{p-1}\right], x\right] .
\end{aligned}
$$

Since $c \in C^{\prime}$ and $x \in \gamma_{n}^{p} A$, one has $\left[c, x^{i}\right] \in\left[C^{\prime}, \gamma_{n}^{p} A\right] \subset \gamma_{n+1}^{p} A$ for all $i, 1 \leq i \leq p-1$, which leads to

$$
[c, x]^{p} \in\left(\gamma_{n+1}^{p} A\right)^{p} \subset \gamma_{n+2}^{p} A \quad \text { and } \quad\left[\left[c, x^{i}\right], x\right] \in\left[\gamma_{n+1}^{p} A, A\right] \subset \gamma_{n+2}^{p} A .
$$

Now, we suppose that $\left[\gamma_{m}^{p} C^{\prime}, \gamma_{n}^{p} A\right] \subset \gamma_{m+n}^{p} A$ for some $m \geq 1$ and all $n \geq 1$ and prove that $\left[\gamma_{m+1}^{p} C^{\prime}, \gamma_{n}^{p} A\right] \subset \gamma_{m+n+1}^{p} A$. As above, there are two cases which work in the same way: 
(i)

$$
\begin{aligned}
{\left[\left[C^{\prime}, \gamma_{m}^{p} C^{\prime}\right], \gamma_{n}^{p} A\right] } & =\left[\left[\gamma_{n}^{p} A, C^{\prime}\right], \gamma_{m}^{p} C^{\prime}\right]\left[\left[\gamma_{m}^{p} C^{\prime}, \gamma_{n}^{p} A\right], C^{\prime}\right] \\
& \subset\left[\gamma_{n+1}^{p} A, \gamma_{m}^{p} C^{\prime}\right]\left[\gamma_{m+n}^{p} A, C^{\prime}\right] \\
& \subset \gamma_{m+n+1}^{p} A .
\end{aligned}
$$

(ii) For $c \in \gamma_{m}^{p} C^{\prime}$ and $x \in \gamma_{n}^{p} A$, one has

$$
\left[c^{p}, x\right]=\left[c,\left[x, c^{p-1}\right]\right]\left[c^{p-1}, x\right][c, x]=\cdots=\left[c,\left[x, c^{p-1}\right]\right] \cdots[c,[x, c]][c, x]^{p},
$$

which is an element of $\gamma_{m+n+1}^{p} A$ by induction hypotheses.

Proof of Theorem 3.3 The restrictions of $\lambda$ and $\sigma$ give rise to morphisms

$$
\lambda_{n}: \gamma_{n}^{p} B \rightarrow \gamma_{n}^{p} C \quad \text { and } \quad \sigma_{n}: \gamma_{n}^{p} C \rightarrow \gamma_{n}^{p} B
$$

such that $\lambda_{n} \circ \sigma_{n}=\operatorname{Id}_{\gamma_{n}^{p} C}, \lambda_{n}$ is onto and $\operatorname{Ker}\left(\lambda_{n}\right)=A \cap \gamma_{n}^{p} B$. Thus, we need to prove that $A \cap \gamma_{n}^{p} B=\gamma_{n}^{p} A$ for all $n \geq 1$. Clearly one has $\gamma_{n}^{p} A \subset A \cap \gamma_{n}^{p} B$. In order to prove the reverse inclusion, we follow the method developed in [12] for almost semi-direct products and define $\tau: B \rightarrow B$ by $\tau(b)=(\sigma \lambda(b))^{-1} b$. This map has the following properties:

(i) Since $\lambda \sigma=\operatorname{Id}_{C}, \tau(B) \subset A$.

(ii) For $x \in B, \tau(x)=x$ if and only if $x \in A$.

(iii) For $\left(b_{1}, b_{2}\right) \in B^{2}, \tau\left(b_{1} b_{2}\right)=\left[\sigma \lambda\left(b_{2}\right), \tau\left(b_{1}\right)^{-1}\right] \tau\left(b_{1}\right) \tau\left(b_{2}\right)$.

(iv) For $b \in B$, setting $a=\tau(b)$ and $c=\sigma \lambda(b)$, we get a unique decomposition $b=c a$ with $c \in C^{\prime}=\sigma(C)$ and $a \in A$.

We claim that $\tau\left(\gamma_{n}^{p} B\right) \subset \gamma_{n}^{p} A$ for all $n \geq 1$. From this, we easily conclude the proof: if $x \in A \cap \gamma_{n}^{p} B$, then $x=\tau(x) \in \gamma_{n}^{p} A$.

One has $\tau\left(\gamma_{1}^{p} B\right) \subset \gamma_{1}^{p} A$. Suppose inductively that $\tau\left(\gamma_{n}^{p} B\right) \subset \gamma_{n}^{p} A$ for some $n \geq 1$, and let us prove that $\tau\left(\gamma_{n+1}^{p} B\right) \subset \gamma_{n+1}^{p} A$. Suppose first that $x$ is an element of $\gamma_{n}^{p} B$. Then using (iii) we get

$$
\begin{aligned}
\tau\left(x^{p}\right) & =\left[\sigma \lambda(x), \tau\left(x^{p-1}\right)^{-1}\right] \tau\left(x^{p-1}\right) \tau(x) \\
& \vdots \\
& =\left[\sigma \lambda(x), \tau\left(x^{p-1}\right)^{-1}\right]\left[\sigma \lambda(x), \tau\left(x^{p-2}\right)^{-1}\right] \cdots\left[\sigma \lambda(x), \tau(x)^{-1}\right] \tau(x)^{p} .
\end{aligned}
$$

Since $\sigma \lambda(x) \in \gamma_{n}^{p} C^{\prime}$, and since $\tau\left(x^{i}\right) \in \gamma_{n}^{p} A$ for $1 \leq i \leq p-1$ by the induction hypothesis, we get

$$
\tau\left(x^{p}\right) \in\left[\gamma_{n}^{p} C^{\prime}, \gamma_{n}^{p} A\right] \cdot\left(\gamma_{n}^{p} A\right)^{p} \subset \gamma_{n+1}^{p} A
$$


by Lemma 3.4: this proves that $\tau\left(\left(\gamma_{n}^{p} B\right)^{p}\right) \subset \gamma_{n+1}^{p} A$. Next, let $b \in B$ and $x \in \gamma_{n}^{p} B$. Setting $a=\tau(b) \in A, y=\tau(x) \in \gamma_{n}^{p} A$ by the induction hypothesis, $c=\sigma \lambda(b) \in C^{\prime}$ and $z=\sigma \lambda(x) \in \gamma_{n}^{p} C^{\prime}$, we get

$$
\begin{aligned}
\tau([b, x]) & =(\sigma \lambda([b, x]))^{-1}[b, x] \\
& =[\sigma \lambda(b), \sigma \lambda(x)]^{-1}[b, x] \\
& =[c, z]^{-1}[c a, z y]=[z, c] a^{-1} c^{-1} y^{-1} z^{-1} c a z y \\
& =[z, c]\left(a^{-1} c^{-1} y^{-1} c y a\right)\left(a^{-1} y^{-1} c^{-1} z^{-1} c z y a\right)\left(a^{-1} y^{-1} z^{-1} a z y\right) \\
& =[z, c]\left(a^{-1}[c, y] a\right)\left(a^{-1} y^{-1}[c, z] y a\right)\left(a^{-1} y^{-1} a y\right)\left(y^{-1} a^{-1} z^{-1} a z y\right) \\
& =[z, c]\left(a^{-1}[c, y] a\right)\left(a^{-1} y^{-1}[c, z] y a\right)[a, y]\left(y^{-1}[a, z] y\right) \\
& =\left[[c, z],\left(a^{-1}[y, c] a\right)\right]\left(a^{-1}[c, y] a\right)[z, c]\left(a^{-1} y^{-1}[c, z] y a\right)[a, y]\left(y^{-1}[a, z] y\right) \\
& =\left[[c, z],\left(a^{-1}[y, c] a\right)\right]\left(a^{-1}[c, y] a\right)[[c, z], y a][a, y]\left(y^{-1}[a, z] y\right) .
\end{aligned}
$$

Now,

$$
[c, z] \in\left[C^{\prime}, \gamma_{n}^{p} C^{\prime}\right] \subset \gamma_{n+1}^{p} C^{\prime} \quad \text { and } \quad[y, c] \in\left[\gamma_{n}^{p} A, C^{\prime}\right] \subset \gamma_{n+1}^{p} A
$$

(Lemma 3.4), thus $\left[[c, z],\left(a^{-1}[y, c] a\right)\right] \in \gamma_{n+1}^{p} A$. Then

$$
\begin{aligned}
{[[c, z], y a] } & \in\left[\gamma_{n+1}^{p} C^{\prime}, A\right] \subset \gamma_{n+1}^{p} A, \\
{[a, y] } & \in\left[A, \gamma_{n}^{p} A\right] \subset \gamma_{n+1}^{p} A, \\
{[a, z] } & \in\left[A, \gamma_{n}^{p} C^{\prime}\right] \subset \gamma_{n+1}^{p} A .
\end{aligned}
$$

Thus, $\tau([b, x]) \in \gamma_{n+1}^{p} A$ and $\tau\left(\left[B, \gamma_{n}^{p} B\right]\right) \subset \gamma_{n+1}^{p} A$.

\section{Corollary 3.5 Let}

$$
1 \longrightarrow A \hookrightarrow B \underset{\sigma \ldots \ldots}{\longrightarrow} C \longrightarrow 1
$$

be a split exact sequence such that $B$ is a $p$-almost direct product of $A$ and $C$. If $A$ and $C$ are residually $p$-finite, then $B$ is residually $p$-finite.

\subsection{Augmentation ideals}

Given a group $G$ and $\mathbb{K}=\mathbb{Z}$ or $\mathbb{F}_{2}$, we will denote by $\mathbb{K}[G]$ the group ring of $G$ over $\mathbb{K}$ and by $\mathbb{K}[G]_{j}$ the augmentation ideal of $G$. The group ring $\mathbb{K}[G]$ is filtered by the powers $\overline{\mathbb{K}}[G]^{j}$ of $\overline{\mathbb{K}[G]}$, and we can define the associated graded algebra

$$
\operatorname{gr} \mathbb{K}[G]=\bigoplus \overline{\mathbb{K}}[G]^{j} / \overline{\mathbb{K}[G]}^{j+1} \text {. }
$$


The following theorem provides a decomposition formula for the augmentation ideal of a 2-almost direct product (see [25, Theorem 3.1] for an analogous result in the case of almost direct products).

Let $A \rtimes C$ be a semi-direct product between two groups $A$ and $C$. It is a classical result that the map $a \otimes c \mapsto a c$ induces a $\mathbb{K}$-isomorphism from $\mathbb{K}[A] \otimes \mathbb{K}[C]$ to $\mathbb{K}[A \rtimes C]$. Identifying these two $\mathbb{K}$-modules, we have the following:

Theorem 3.6 If $A \rtimes C$ is a 2-almost direct product, then

$$
{\overline{\mathrm{F}_{2}[A \rtimes C]}}^{k}=\sum_{i+h=k}{\overline{\mathrm{F}_{2}[A]}}^{i} \otimes{\overline{\mathrm{F}_{2}[C]}}^{h} \text { for all } k .
$$

Proof We sketch the proof, which is almost verbatim the same as the proof of [25, Theorem 3.1]. Let

$$
R_{k}=\sum_{i+h=k}{\overline{\mathrm{F}_{2}[A]}}^{i} \otimes{\overline{\mathrm{F}_{2}[C]}}^{h}
$$

$R_{k}$ is a descending filtration on $\mathbb{F}_{2}[A] \otimes \mathbb{F}_{2}[C]$, and with the above identification, we get that $R_{k} \subset{\overline{\mathbb{F}_{2}[A \rtimes C]}}^{k}$. To verify the other inclusion we have to check that $\prod_{j=1}^{k}\left(a_{j} c_{j}-1\right) \in R_{k}$ for every $a_{1}, \ldots, a_{k}$ in $A$ and $c_{1}, \ldots, c_{k}$ in $C$. Actually it is enough to verify that $e=\prod_{j=1}^{k}\left(e_{j}-1\right) \in R_{k}$ where either $e_{j} \in A$ or $e_{j} \in C$ (see [25, Theorem 3.1] for a proof of this fact); we call $e$ a special element. We associate to a special element $e$ an element in $\{0,1\}^{k}$ : let type $(e)=\left(\delta\left(e_{1}\right), \ldots, \delta\left(e_{k}\right)\right)$, where $\delta\left(e_{j}\right)=0$ if $e_{j} \in A$ and $\delta\left(e_{j}\right)=1$ if $e_{j} \in C$. We will say that the special element $e$ is standard if

$$
\operatorname{type}(e)=(\overbrace{0, \ldots, 0}^{i}, \overbrace{1, \ldots, 1}^{h}) .
$$

In this case $e \in{\overline{\mathbb{F}_{2}[A]}}^{i} \otimes{\overline{\mathbb{F}_{2}[C]}}^{h} \subset R_{k}$ and we are done. We claim that we can reduce all special elements to linear combinations of standard elements. If $e$ is not standard, then it must be of the form

$$
e=\prod_{i=1}^{r}\left(a_{i}-1\right) \prod_{j=1}^{s}\left(c_{j}-1\right)(c-1)(a-1) \prod_{l=1}^{t}\left(e_{l}-1\right),
$$

where $a_{1}, \ldots, a_{r}, a \in A, c_{1}, \ldots, c_{s}, c \in C$, the element $\tilde{e}=\prod_{l=1}^{t}\left(e_{l}-1\right)$ is special and $r+s+t+2=k$. Therefore

$$
\operatorname{type}(e)=(\overbrace{0, \ldots, 0}^{r}, \overbrace{1, \ldots, 1}^{s}, 1,0, \delta\left(e_{1}\right), \ldots, \delta\left(e_{t}\right)) .
$$


Now we can use the assumption that $A \rtimes C$ is a 2-almost direct product to claim that one has commutation relations in $\mathbb{Z}[A \rtimes C]$ expressing the difference

$$
(c-1)(a-1)-(a-1)(c-1)
$$

as a linear combination of terms of the form

$$
\left(a^{\prime}-1\right)\left(a^{\prime \prime}-1\right) c \quad \text { with } a^{\prime}, a^{\prime \prime} \in A,
$$

for any $a \in A$ and $c \in C$. In fact,

$$
(c-1)(a-1)-(a-1)(c-1)=c a-a c=\left(c a c^{-1} a^{-1}-1\right) a c=(f-1) a c,
$$

where $f=\left[c^{-1}, a^{-1}\right] \in[C, A] \subset \gamma_{2}^{2}(A)$ by Lemma 3.4. We can decompose $f$ as $f=h_{1} k_{1} \cdots h_{m} k_{m}$, where, for $j=1, \ldots, m, h_{j}$ belongs to $[A, A]$ and $k_{j}=\left(k_{j}^{\prime}\right)^{2}$ for some $k_{j}^{\prime} \in A$. One knows (see for instance [10, page 194]) that, for $j=1, \ldots, m$, $\left(h_{j}-1\right)$ is a linear combination of terms of the form

$$
\left(h_{j}^{\prime}-1\right)\left(h_{j}^{\prime \prime}-1\right) \alpha_{j} \quad \text { with } h_{j}^{\prime}, h_{j}^{\prime \prime}, \alpha_{j} \in A .
$$

On the other hand, for $j=1, \ldots, m$, we have also that

$$
\left(k_{j}-1\right)=\left(k_{j}^{\prime}-1\right)\left(k_{j}^{\prime}-1\right) \quad \text { with } k_{j}^{\prime} \in A, \quad \text { since the coefficients are in } \mathbb{F}_{2} .
$$

Then, recalling that $(h k-1)=(h-1) k+(k-1)$ for any $h, k \in A$, we can conclude that $f-1$ can be rewritten as a linear combination of terms of the form

$$
\left(f^{\prime}-1\right)\left(f^{\prime \prime}-1\right) \alpha \text { with } f^{\prime}, f^{\prime \prime}, \alpha \in A
$$

and that $(c-1)(a-1)-(a-1)(c-1)$ is a linear combination of terms of the form

$$
\left(f^{\prime}-1\right)\left(f^{\prime \prime}-1\right) \alpha c \text { with } f^{\prime}, f^{\prime \prime}, \alpha \in A .
$$

Rewriting $\left(f^{\prime \prime}-1\right) \alpha$ as $\left(f^{\prime \prime} \alpha-1\right)-(\alpha-1)$, we obtain that the difference

$$
(c-1)(a-1)-(a-1)(c-1)
$$

can be seen as a linear combination of terms of the form

$$
\left(a^{\prime}-1\right)\left(a^{\prime \prime}-1\right) c \text { with } a^{\prime}, a^{\prime \prime} \in A .
$$

Therefore $e$ can be rewritten as a sum whose first term is the special element

$$
e^{\prime}=\prod_{i=1}^{r}\left(a_{i}-1\right) \prod_{j=1}^{s}\left(c_{j}-1\right)(a-1)(c-1) \prod_{l=1}^{t}\left(e_{l}-1\right)
$$


and whose second term is a linear combination of elements of the form $e^{\prime \prime} c$, where

$$
e^{\prime \prime}=\prod_{i=1}^{r}\left(a_{i}-1\right) \prod_{j=1}^{s}\left(c_{j}-1\right)\left(a^{\prime}-1\right)\left(a^{\prime \prime}-1\right) \prod_{l=1}^{t}\left(c e_{l} c^{-1}-1\right) .
$$

Using the lexicographic order from the left, one has type $(e)>\operatorname{type}\left(e^{\prime}\right)$ and type $(e)>$ type $\left(e^{\prime \prime}\right)$.

By induction on the lexicographic order, we infer that $e^{\prime}$ and $e^{\prime \prime}$ belong to $R_{k}$; since $R_{k} \cdot c \subset R_{k}$ for any $c \in C$, it follows that $e$ belongs to $R_{k}$ and we are done.

\section{The closed case}

\subsection{A presentation of $P_{n}\left(N_{g}\right)$ and induced identities}

We recall a group presentation of $P_{n}\left(N_{g}\right)$ given in [16]; the geometric interpretation of generators is provided in Figure 4.

Theorem 4.1 [16] For $g \geq 2$ and $n \geq 1, P_{n}\left(N_{g}\right)$ has a presentation with generators

$$
\left(B_{i, j}\right)_{1 \leq i<j \leq n} \quad \text { and } \quad\left(\rho_{k, l}\right)_{\substack{1 \leq k \leq n \\ 1 \leq l \leq g}}
$$

and relations of the following four types:

(a) For $1 \leq i<j \leq n$ and $1 \leq r<s \leq n$,

$B_{r, s} B_{i, j} B_{r, s}^{-1}$

$$
= \begin{cases}B_{i, j} & \text { if } i<r<s<j \text { or } r<s<i<j, \\ B_{i, j}^{-1} B_{r, j}^{-1} B_{i, j} B_{r, j} B_{i, j} & \text { if } r<i=s<j, \\ B_{s, j}^{-1} B_{i, j} B_{s, j} & \text { if } i=r<s<j \\ B_{s, j}^{-1} B_{r, j}^{-1} B_{s, j} B_{r, j} B_{i, j} B_{r, j}^{-1} B_{s, j}^{-1} B_{r, j} B_{s, j} & \text { if } r<i<s<j .\end{cases}
$$

(b) For $1 \leq i<j \leq n$ and $1 \leq k, l \leq g$,

$$
\rho_{i, k} \rho_{j, l} \rho_{i, k}^{-1}= \begin{cases}\rho_{j, l} & \text { if } k<l . \\ \rho_{j, k}^{-1} B_{i, j}^{-1} \rho_{j, k}^{2} & \text { if } k=l . \\ \rho_{j, k}^{-1} B_{i, j}^{-1} \rho_{j, k} B_{i, j}^{-1} \rho_{j, l} B_{i, j} \rho_{j, k}^{-1} B_{i, j} \rho_{j, k} & \text { if } k>l .\end{cases}
$$

(c) For $1 \leq i \leq n$,

$$
\rho_{i, 1}^{2} \cdots \rho_{i, g}^{2}=T_{i}, \quad \text { where } T_{i}=B_{1, i} \cdots B_{i-1, i} B_{i, i+1} \cdots B_{i, n} .
$$


(d) For $1 \leq i<j \leq n, 1 \leq k \leq n, k \neq j$ and $1 \leq l \leq g$,

$\rho_{k, l} B_{i, j} \rho_{k, l}^{-1}= \begin{cases}B_{i, j} & \text { if } k<i \text { or } j<k, \\ \rho_{j, l}^{-1} B_{i, j}^{-1} \rho_{j, l} & \text { if } k=i, \\ \rho_{j, l}^{-1} B_{k, j}^{-1} \rho_{j, l} B_{k, j}^{-1} B_{i, j} B_{k, j} \rho_{j, l}^{-1} B_{k, j} \rho_{j, l} l & \text { if } i<k<j .\end{cases}$

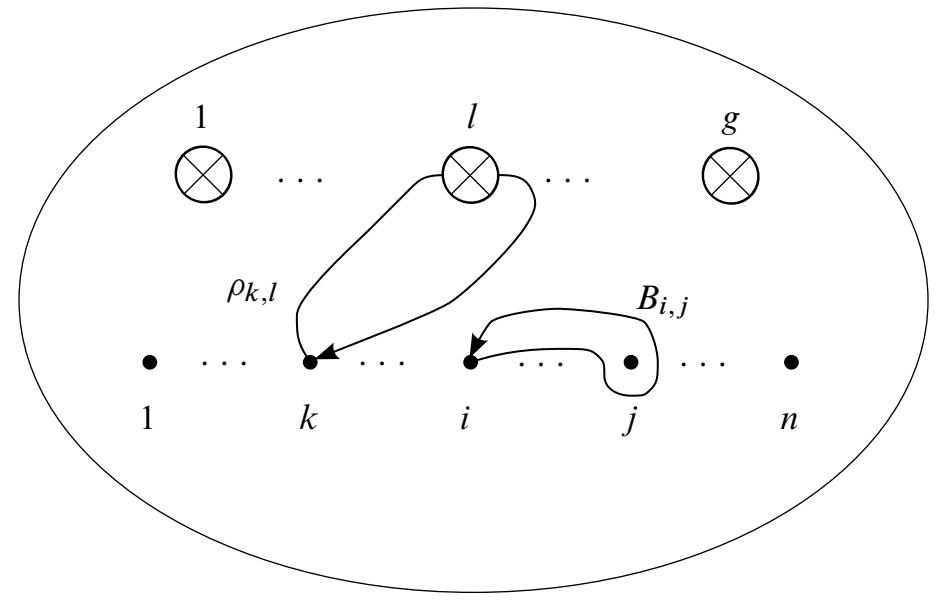

Figure 4: Generators of $P_{n}\left(N_{g}\right)$.

Let us denote by $U$ the element $\rho_{n, 1} \rho_{n-1,1} \cdots \rho_{2,1}$ of $P_{n}\left(N_{g}\right)$.

Lemma 4.2 The following relations hold in $P_{n}\left(N_{g}\right)$ :

(1) $\left[\rho_{i, k}, \rho_{j, k}^{-1}\right]=B_{i, j}^{-1}$ for $1 \leq i<j \leq n$ and $1 \leq k \leq g$.

(2) $\left[\rho_{k, 1}, \rho_{1,1} \rho_{1,1} \rho_{1,2} T_{1}^{-1}\right]=1$ for $2 \leq k \leq n$.

(3) $\left[U, \rho_{1,1} \rho_{1,1} \rho_{1,2} T_{1}^{-1}\right]=1$.

(4) $U \rho_{1,1} U^{-1}=\rho_{1,1} T_{1}^{-1}$.

Proof The first and second identities can be verified by drawing the corresponding braids (see Figure 5 and 6). The third one is a direct consequence of the second one and the definition of $U$. We prove the last one as follows:

$$
\begin{aligned}
\rho_{1,1}^{-1} U \rho_{1,1} & =\left(\rho_{1,1}^{-1} \rho_{n, 1} \rho_{1,1}\right) \cdots\left(\rho_{1,1}^{-1} \rho_{2,1} \rho_{1,1}\right) \\
& =\left(B_{1, n}^{-1} \rho_{n, 1}\right) \cdots\left(B_{1,2}^{-1} \rho_{2,1}\right) \quad \text { by }(\mathrm{e}) \\
& =B_{1, n}^{-1} \cdots B_{1,2}^{-1} \rho_{n, 1} \cdots \rho_{2,1} \quad \text { by }\left(\mathrm{d}_{1}\right) \\
& =T_{1}^{-1} U .
\end{aligned}
$$



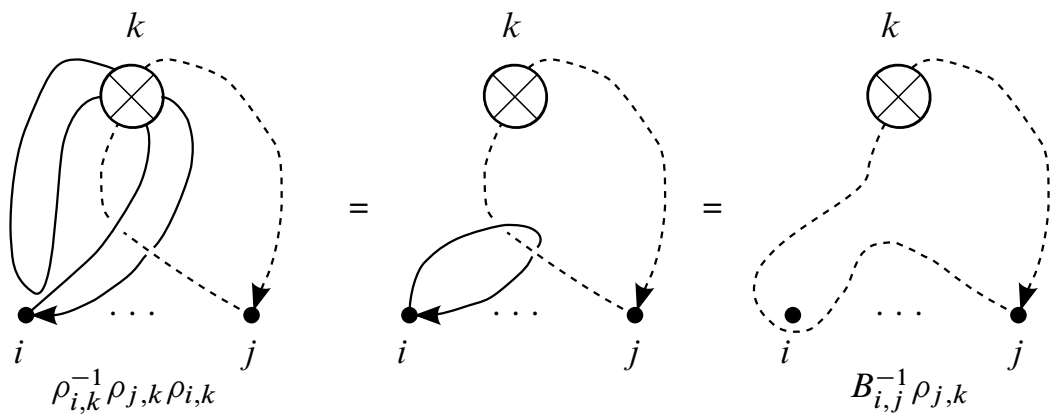

Figure 5: Identity (e).

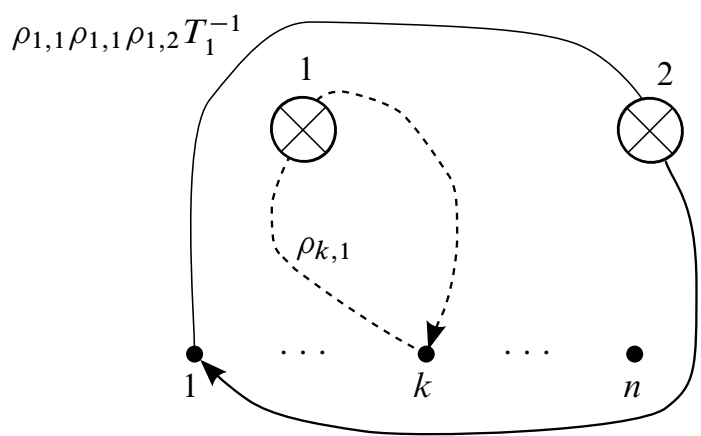

Figure 6: Identity (f).

\subsection{The pure braid group $P_{n}\left(N_{g}\right)$ is residually 2-finite}

Following [14], one has, for $g \geq 2$, a split exact sequence

$$
1 \longrightarrow P_{n-1}\left(N_{g, 1}\right) \stackrel{\mu}{\longrightarrow} P_{n}\left(N_{g}\right) \stackrel{\lambda}{\longrightarrow} P_{1}\left(N_{g}\right)=\pi_{1}\left(N_{g}\right) \longrightarrow 1,
$$

where $\lambda$ is induced by the map which forgets all strands except the first one, and $\mu$ is defined by capping the boundary component by a disc with one marked point (the first strand in $P_{n}\left(N_{g}\right)$ ). According to the definition of $\mu$ and to Proposition 2.3, $\operatorname{Im}(\mu)$ is generated by $\left\{\rho_{i, k} \mid 2 \leq i \leq n, 1 \leq k \leq g\right\} \cup\left\{B_{i, j} \mid 2 \leq i<j \leq n\right\}$.

The section given in [14] is geometric, ie it is induced by a crossed section at the level of fibrations. In order to study the action of $\pi_{1}\left(N_{g}\right)$ on $P_{n-1}\left(N_{g, 1}\right)$, we need an algebraic section. Recall that $\pi_{1}\left(N_{g}\right)$ has a group presentation with generators $p_{1}, \ldots, p_{g}$ and the single relation $p_{1}^{2} \cdots p_{g}^{2}=1$. We define the set map $\sigma: \pi_{1}\left(N_{g}\right) \rightarrow P_{n}\left(N_{g}\right)$ by setting

$$
\sigma\left(p_{i}\right)= \begin{cases}T_{1}^{-1} U \rho_{1,1} T_{1} & \text { for } i=1, \\ T_{1}^{-1} \rho_{1,1}^{-1} U^{-1} \rho_{1,1} \rho_{1,2} & \text { for } i=2, \\ \rho_{1, i} & \text { for } 3 \leq i \leq g .\end{cases}
$$


Proposition 4.3 The map $\sigma$ is a well-defined homomorphism satisfying $\lambda \circ \sigma=$ $\operatorname{Id}_{\pi_{1}\left(N_{g}\right)}$.

Proof Since $\lambda\left(\rho_{1, i}\right)=p_{i}$ for all $1 \leq i \leq g$ and $\lambda(U)=\lambda\left(T_{1}\right)=1$, one clearly has $\lambda \sigma=\operatorname{Id}_{\pi_{1}\left(N_{g}\right)}$ if $\sigma$ is a group homomorphism. Thus, we just have to prove that $\sigma\left(p_{1}\right)^{2} \cdots \sigma\left(p_{g}\right)^{2}=1$ :

$$
\begin{aligned}
\sigma\left(p_{1}\right)^{2} \cdots \sigma\left(p_{g}\right)^{2}= & T_{1}^{-1} U \rho_{1,1} T_{1} T_{1}^{-1} U \rho_{1,1} T_{1} T_{1}^{-1} \rho_{1,1}^{-1} U^{-1} \rho_{1,1} \rho_{1,2} T_{1}^{-1} \\
& \times \rho_{1,1}^{-1} U^{-1} \rho_{1,1} \rho_{1,2} \rho_{1,3}^{2} \cdots \rho_{1, g}^{2} \\
= & T_{1}^{-1} U \rho_{1,1} \rho_{1,1} \rho_{1,2} T_{1}^{-1} \rho_{1,1}^{-1} U^{-1} \rho_{1,1} \rho_{1,2} \rho_{1,3}^{2} \cdots \rho_{1, g}^{2} \\
= & T_{1}^{-1} \rho_{1,1} \rho_{1,1} \rho_{1,2} T_{1}^{-1} U \rho_{1,1}^{-1} U^{-1} \rho_{1,1} \rho_{1,2} \rho_{1,3}^{2} \cdots \rho_{1, g}^{2} \quad \text { by (g) } \\
= & T_{1}^{-1} \rho_{1,1} \rho_{1,1} \rho_{1,2} T_{1}^{-1} T_{1} \rho_{1,1}^{-1} \rho_{1,1} \rho_{1,2} \rho_{1,3}^{2} \cdots \rho_{1, g}^{2} \quad \text { by (h) } \\
= & T_{1}^{-1} \rho_{1,1} \rho_{1,1} \rho_{1,2} \rho_{1,2} \rho_{1,3}^{2} \cdots \rho_{1, g}^{2} \\
= & 1 \quad \text { by (c). }
\end{aligned}
$$

So, the exact sequence (1) splits. In order to apply Theorem 3.3, we have to prove that the action of $\pi_{1}\left(N_{g}\right)$ on $P_{n-1}\left(N_{g, 1}\right)$ is trivial on $H_{1}\left(P_{n-1}\left(N_{g, 1}\right) ; \mathbb{F}_{2}\right)$. This is the claim of the following proposition.

Proposition 4.4 For all $x \in \operatorname{Im}(\sigma)$ and $a \in \operatorname{Im}(\mu)$, one has

$$
\left[x^{-1}, a^{-1}\right]=x a x^{-1} a^{-1} \in \gamma_{2}^{2}(\operatorname{Im}(\mu)) .
$$

Proof It is enough to prove the result for generators

$$
a \in\left\{B_{j, k} \mid 2 \leq j<k \leq n\right\} \cup\left\{\rho_{j, l} \mid 2 \leq j \leq n \text { and } 1 \leq l \leq g\right\}
$$

and

$$
x \in\left\{\sigma\left(p_{1}\right), \ldots, \sigma\left(p_{g}\right)\right\}
$$

of $\operatorname{Im}(\mu)$ and $\operatorname{Im}(\sigma)$, respectively. Suppose first that $2 \leq j<k \leq n$. One has:

- $\left[\sigma\left(p_{i}\right)^{-1}, B_{j, k}^{-1}\right]=\left[\rho_{1, i}^{-1}, B_{j, k}^{-1}\right]=1$ for $3 \leq i \leq g$ by $\left(\mathrm{d}_{1}\right)$.

- Then, one has

$$
\begin{aligned}
{\left[\sigma\left(p_{2}\right)^{-1}, B_{j, k}^{-1}\right] } & =\left[\rho_{1,2}^{-1} \rho_{1,1}^{-1} U \rho_{1,1} T_{1}, B_{j, k}^{-1}\right] \\
& =\left(\rho_{1,1}^{-1} U \rho_{1,1} T_{1}\right)^{-1}\left[\rho_{1,2}^{-1}, B_{j, k}^{-1}\right]\left(\rho_{1,1}^{-1} U \rho_{1,1} T_{1}\right)\left[\rho_{1,1}^{-1} U \rho_{1,1} T_{1}, B_{j, k}^{-1}\right] \\
& =\left[\rho_{1,1}^{-1} U \rho_{1,1} T_{1}, B_{j, k}^{-1}\right] \quad \text { by }\left(\mathrm{d}_{1}\right) .
\end{aligned}
$$


But $U$ and $T_{1}$ are elements of $\operatorname{Im}(\mu)$ and the latter being normal in $P_{n}\left(N_{g}\right)$, $\rho_{1,1}^{-1} U \rho_{1,1} T_{1} \in \operatorname{Im}(\mu)$ thus $\left[\sigma\left(p_{2}\right)^{-1}, B_{j, k}^{-1}\right] \in \Gamma_{2}(\operatorname{Im}(\mu)) \subset \gamma_{2}^{2}(\operatorname{Im}(\mu))$.

- In the same way, one has

$$
\begin{aligned}
& {\left[\sigma\left(p_{1}\right)^{-1}, B_{j, k}^{-1}\right]=\left[T_{1}^{-1} \rho_{1,1}^{-1} U^{-1} T_{1}, B_{j, k}^{-1}\right]=\left[\rho_{1,1}^{-1} \rho_{1,1} T_{1}^{-1} \rho_{1,1}^{-1} U^{-1} T_{1}, B_{j, k}^{-1}\right]} \\
& =\left(\rho_{1,1} T_{1}^{-1} \rho_{1,1}^{-1} U^{-1} T_{1}\right)^{-1}\left[\rho_{1,1}^{-1}, B_{j, k}^{-1}\right]\left(\rho_{1,1} T_{1}^{-1} \rho_{1,1}^{-1} U^{-1} T_{1}\right) \\
& \times\left[\rho_{1,1} T_{1}^{-1} \rho_{1,1}^{-1} U^{-1} T_{1}, B_{j, k}^{-1}\right] \\
& =\left[\rho_{1,1} T_{1}^{-1} \rho_{1,1}^{-1} U^{-1} T_{1}, B_{j, k}^{-1}\right] \text { by }\left(\mathrm{d}_{1}\right) \text {, }
\end{aligned}
$$

thus, as before, $\left[\sigma\left(p_{1}\right)^{-1}, B_{j, k}^{-1}\right] \in \gamma_{2}^{2}(\operatorname{Im}(\mu))$.

Now, let $j$ and $l$ be integers such that $2 \leq j \leq n$ and $1 \leq l \leq g$, and let us first prove that $\left[\rho_{1, i}^{-1}, \rho_{j, l}^{-1}\right] \in \gamma_{2}^{2}(\operatorname{Im}(\mu))$ for all $i, 1 \leq i \leq g$ :

- This is clear for $i<l$ by $\left(\mathrm{b}_{1}\right)$.

- For $i=l$, the relation $\left(\mathrm{b}_{2}\right)$ gives $\left[\rho_{1, l}^{-1}, \rho_{j, l}^{-1}\right]=\rho_{j, l}^{-1} B_{1, j}^{-1} \rho_{j, l}$. But

$$
B_{1, j}^{-1}=B_{2, j} \cdots B_{j-1, j} B_{j, j+1} \cdots B_{j, n} \rho_{j, g}^{-2} \cdots \rho_{j, 1}^{-2} \quad \text { (relation (c)) }
$$

is an element of $\gamma_{2}^{2}(\operatorname{Im}(\mu))$ by (e), thus we get $\left[\rho_{1, l}^{-1}, \rho_{j, l}^{-1}\right] \in \gamma_{2}^{2}(\operatorname{Im}(\mu))$.

- If $l<i$ then $\left[\rho_{1, i}^{-1}, \rho_{j, l}^{-1}\right]=\left[B_{1, j} \rho_{j, i}^{-1} B_{1, j} \rho_{j, i}, \rho_{j, l}^{-1}\right]$ by $\left(\mathrm{b}_{3}\right)$ so $\left[\rho_{1, i}^{-1}, \rho_{j, l}^{-1}\right] \in$ $\gamma_{2}^{2}(\operatorname{Im}(\mu))$ since $\rho_{j, l}, \rho_{j, i}$ and $B_{1, j}$ are elements of $\operatorname{Im}(\mu)$.

From this, we deduce the following facts.

(1) $\left[\sigma\left(p_{i}\right)^{-1}, \rho_{j, l}^{-1}\right] \in \gamma_{2}^{2}(\operatorname{Im}(\mu))$ for $i \geq 3$ since $\sigma\left(p_{i}\right)=\rho_{1, i}$.

(2) Next, one has

$$
\begin{aligned}
{\left[\sigma\left(p_{2}\right)^{-1}, \rho_{j, l}^{-1}\right] } & =\left[\rho_{1,2}^{-1} \rho_{1,1}^{-1} U \rho_{1,1} T_{1}, \rho_{j, l}^{-1}\right] \\
& =\left(\rho_{1,1}^{-1} U \rho_{1,1} T_{1}\right)^{-1}\left[\rho_{1,2}^{-1}, \rho_{j, l}^{-1}\right]\left(\rho_{1,1}^{-1} U \rho_{1,1} T_{1}\right)\left[\rho_{1,1}^{-1} U \rho_{1,1} T_{1}, \rho_{j, l}^{-1}\right] .
\end{aligned}
$$

Since $\rho_{1,1}^{-1} U \rho_{1,1} T_{1}$ and $\rho_{j, l}$ are elements of $\operatorname{Im}(\mu)$ and $\left[\rho_{1,2}^{-1}, \rho_{j, l}^{-1}\right]$ is an element of $\gamma_{2}^{2}(\operatorname{Im}(\mu))$, we get $\left[\sigma\left(p_{2}\right)^{-1}, \rho_{j, l}^{-1}\right] \in \gamma_{2}^{2}(\operatorname{Im}(\mu))$.

(3) In the same way, one has

$$
\begin{aligned}
{\left[\sigma\left(p_{1}\right)^{-1}, \rho_{j, l}^{-1}\right] } & =\left[T_{1}^{-1} \rho_{1,1}^{-1} U^{-1} T_{1}, \rho_{j, l}^{-1}\right]=\left[T_{1}^{-1} \rho_{1,1}^{-1} U^{-1} T_{1} \rho_{1,1} \rho_{1,1}^{-1}, \rho_{j, l}^{-1}\right] \\
& =\rho_{1,1}\left[T_{1}^{-1} \rho_{1,1}^{-1} U^{-1} T_{1} \rho_{1,1}, \rho_{j, l}^{-1}\right] \rho_{1,1}^{-1}\left[\rho_{1,1}^{-1}, \rho_{j, l}^{-1}\right] \in \gamma_{2}^{2}(\operatorname{Im}(\mu)) .
\end{aligned}
$$


We are now ready to prove the main result of this section.

Theorem 4.5 For all $g \geq 2$ and $n \geq 1$, the pure braid group $P_{n}\left(N_{g}\right)$ is residually 2-finite.

Proof Proposition 4.3 says that the sequence

$$
1 \longrightarrow P_{n-1}\left(N_{g, 1}\right) \longrightarrow P_{n}\left(N_{g}\right) \longrightarrow \pi_{1}\left(N_{g}\right) \longrightarrow 1
$$

splits. Now $P_{n-1}\left(N_{g, 1}\right)$ is residually 2-finite (Theorem 2.7). It is proved in [2] and [3] that $\pi_{1}\left(N_{g}\right)$ is residually free for $g \geq 4$, so it is residually $2-$ finite. This result is proved in [23, Lemma 8.9] for $g=3$. When $g=2, \pi_{1}\left(N_{2}\right)$ has presentation $\left\langle a, b \mid a b a^{-1}=b^{-1}\right\rangle$ so is a 2 -almost direct product of $\mathbb{Z}$ by $\mathbb{Z}$. Since $\mathbb{Z}$ is residually 2-finite, $\pi_{1}\left(N_{2}\right)$ is residually 2 -finite by Corollary 3.5. So, using Proposition 4.4 and Corollary 3.5, we can conclude that $P_{n}\left(N_{g}\right)$ is residually 2-finite.

\subsection{The case $P_{n}\left(\mathbb{R P}^{2}\right)$}

The main reason to exclude $N_{1}=\mathbb{R P}^{2}$ in Theorem 4.5 is that the exact sequence (1) doesn't exist in this case, but forgetting at most $n-2$ strands we get the following exact sequence ( $1 \leq m \leq n-2$; see [9]):

$$
1 \longrightarrow P_{m}\left(N_{1, n-m}\right) \longrightarrow P_{n}\left(\mathbb{R P}^{2}\right) \longrightarrow P_{n-m}\left(\mathbb{R P}^{2}\right) \longrightarrow 1 .
$$

This sequence splits if and only if $n=3$ and $m=1$ (see [15]). Thus, what we know is the following:

- $\quad P_{1}\left(\mathbb{R} \mathbb{P}^{2}\right)=\pi_{1}\left(\mathbb{R P}^{2}\right)=\mathbb{Z} / 2 \mathbb{Z}: P_{1}\left(\mathbb{R} \mathbb{P}^{2}\right)$ is a 2-group.

- $\quad P_{2}\left(\mathbb{R P}^{2}\right)=Q_{8}$, the quaternion group (see [9]): $P_{2}\left(\mathbb{R P}^{2}\right)$ is a 2-group.

- One has the split exact sequence

$$
1 \longrightarrow P_{1}\left(N_{1,2}\right) \longrightarrow P_{3}\left(\mathbb{R P}^{2}\right) \longrightarrow P_{2}\left(\mathbb{R P}^{2}\right) \longrightarrow 1,
$$

where $P_{1}\left(N_{1,2}\right)=\pi_{1}\left(N_{1,2}\right)$ is a free group of rank 2 , thus is residually $2-$ finite. Since $P_{2}\left(\mathbb{R} P^{2}\right)$ is 2-finite, we can conclude that $P_{3}\left(\mathbb{R P} P^{2}\right)$ is residually 2-finite using [19, Lemma 1.5].

\section{References}

[1] V G Bardakov, P Bellingeri, On residual properties of pure braid groups of closed surfaces, Comm. Algebra 37 (2009) 1481-1490 MR2526317 
[2] B Baumslag, Residually free groups, Proc. London Math. Soc. 17 (1967) 402-418 MR0215903

[3] G Baumslag, On generalised free products, Math. Z. 78 (1962) 423-438 MR0140562

[4] P Bellingeri, On presentations of surface braid groups, J. Algebra 274 (2004) 543-563 MR2043362

[5] P Bellingeri, L Funar, Braids on surfaces and finite type invariants, C. R. Math. Acad. Sci. Paris 338 (2004) 157-162 MR2038286

[6] P Bellingeri, S Gervais, J Guaschi, Lower central series of Artin-Tits and surface braid groups, J. Algebra 319 (2008) 1409-1427 MR2383053

[7] P Bellingeri, E Godelle, J Guaschi, Exact sequences, lower central series and representations of surface braid groups, preprint (2011) arXiv:1106.4982

[8] R Botto Mura, A Rhemtulla, Orderable groups, Lecture Notes in Pure and Applied Mathematics 27, Marcel Dekker, New York (1977) MR0491396

[9] J van Buskirk, Braid groups of compact 2-manifolds with elements of finite order, Trans. Amer. Math. Soc. 122 (1966) 81-97 MR0189013

[10] K-T Chen, Extension of $C^{\infty}$ function algebra by integrals and Malcev completion of $\pi_{1}$, Advances in Math. 23 (1977) 181-210 MR0458461

[11] E Fadell, L Neuwirth, Configuration spaces, Math. Scand. 10 (1962) 111-118 MR0141126

[12] M Falk, R Randell, The lower central series of a fiber-type arrangement, Invent. Math. 82 (1985) 77-88 MR808110

[13] S Garoufalidis, J Levine, Finite type 3-manifold invariants and the structure of the Torelli group, I, Invent. Math. 131 (1998) 541-594 MR1614551

[14] D L Gonçalves, J Guaschi, On the structure of surface pure braid groups, J. Pure Appl. Algebra 186 (2004) 187-218 MR2025598

[15] D L Gonçalves, J Guaschi, The braid groups of the projective plane and the FadellNeuwirth short exact sequence, Geom. Dedicata 130 (2007) 93-107 MR2365780

[16] DL Gonçalves, J Guaschi, Braid groups of non-orientable surfaces and the Fadell-Neuwirth short exact sequence, J. Pure Appl. Algebra 214 (2010) 667-677 MR2577674

[17] J González-Meneses, Ordering pure braid groups on compact, connected surfaces, Pacific J. Math. 203 (2002) 369-378 MR1897904

[18] J González-Meneses, L Paris, Vassiliev invariants for braids on surfaces, Trans. Amer. Math. Soc. 356 (2004) 219-243 MR2020030

[19] K W Gruenberg, Residual properties of infinite soluble groups, Proc. London Math. Soc. 7 (1957) 29-62 MR0087652 
[20] J Guaschi, D Juan-Pineda, A survey of surface braid groups and the lower algebraic $K$-theory of their group rings, from: "Handbook of group actions, Vol. II", (L Ji, A Papadopoulos, S-T Yau, editors), Adv. Lect. Math. 32, Int. Press, Somerville, MA (2015) 23-75 MR3382024

[21] B Hartley, On residually finite p-groups, from: "Convegno sui Gruppi Infiniti, INDAM, Roma, 1973”, Symposia Mathematica 17, Academic Press, London (1976) 225-234 MR0419611

[22] D L Johnson, Presentations of groups, London Math. Soc. Lect. Notes Ser. 22, Cambridge Univ. Press (1976) MR0396763

[23] G Levitt, A Minasyan, Residual properties of automorphism groups of (relatively) hyperbolic groups, Geom. Topol. 18 (2014) 2985-3023 MR3285227

[24] W Magnus, A Karrass, D Solitar, Combinatorial group theory, 2nd edition, Dover, New York (1976) MR0422434

[25] Ş Papadima, The universal finite-type invariant for braids, with integer coefficients, Topology Appl. 118 (2002) 169-185 MR1877723

[26] L Paris, Residual p properties of mapping class groups and surface groups, Trans. Amer. Math. Soc. 361 (2009) 2487-2507 MR2471926

[27] L Paris, B Szepietowski, A presentation for the mapping class group of a nonorientable surface, Bull. Soc. Math. France 143 (2015) 503-566 MR3417732

[28] G P Scott, Braid groups and the group of homeomorphisms of a surface, Proc. Cambridge Philos. Soc. 68 (1970) 605-617 MR0268889

[29] M Stukow, Commensurability of geometric subgroups of mapping class groups, Geom. Dedicata 143 (2009) 117-142 MR2576298

[30] B Szepietowski, Embedding the braid group in mapping class groups, Publ. Mat. 54 (2010) 359-368 MR2675928

[31] B Szepietowski, Crosscap slides and the level 2 mapping class group of a nonorientable surface, Geom. Dedicata 160 (2012) 169-183 MR2970047

[32] H Zieschang, E Vogt, H-D Coldewey, Surfaces and planar discontinuous groups, Lecture Notes in Mathematics 835, Springer, Berlin (1980) MR606743

Laboratoire de Mathématiques Nicolas Oresme, Université de Caen

CNRS UMR 6139, F-14000 Caen, France

Laboratoire de Mathématique Jean Leray, Université de Nantes

CNRS UMR 6629, 2, rue de la Houssinière, BP 92208, F-44322 Cedex 3 Nantes, France paolo.bellingeri@math.unicaen.fr, sylvain.gervais@univ-nantes.fr

http://www.math. unicaen.fr/ bellinge/,

http://www.math.sciences.univ-nantes.fr/ gervais/

Received: 27 January $2015 \quad$ Revised: 7 July 2015 